LAWRENCE LIVERMORE N A T IO N A L LABORATORY

\title{
Optimal Position Estimation for the Automatic Alignment of a High Energy Laser
}

J. V. Candy, W. A. Mcclay, A. A. S. Awwal, S. W. Ferguson

July 28,2004

Journal of the Optical Society of America A 
This document was prepared as an account of work sponsored by an agency of the United States Government. Neither the United States Government nor the University of California nor any of their employees, makes any warranty, express or implied, or assumes any legal liability or responsibility for the accuracy, completeness, or usefulness of any information, apparatus, product, or process disclosed, or represents that its use would not infringe privately owned rights. Reference herein to any specific commercial product, process, or service by trade name, trademark, manufacturer, or otherwise, does not necessarily constitute or imply its endorsement, recommendation, or favoring by the United States Government or the University of California. The views and opinions of authors expressed herein do not necessarily state or reflect those of the United States Government or the University of California, and shall not be used for advertising or product endorsement purposes. 


\title{
Optimal position estimation for the automatic alignment of a high energy laser
}

\author{
J. V. Candy W. Mcclay A. Awwal and W. Ferguson \\ University of California, Lawrence Livermore National Laboratory, P.O. Box 808, L-156, Livermore, CA 94526
}

Received August 1, 2004

The alignment of high energy laser beams for potential fusion experiments demand high precision and accuracy by the underlying positioning algorithms whether it be for actuator control or monitoring the beam line for potential anomalies. This paper discusses the feasibility of employing on-line optimal position estimators in the form of model-based processors to achieve the desired results. Here we discuss the modeling, development, implementation and processing of model-based processors applied to both simulated and actual beam line data.

OCIS codes:070.6020, 140.3300, 120.1880, 040.1520

\section{INTRODUCTION}

High power, tightly focused laser beams are required to achieve successful ignition and therefore fusion at the Lawrence Livermore National Laboratory (LLNL) National Ignition Facility (NIF). ${ }^{1}$ The beams must simultaneously focus precisely on a nanoscale target capsule to succeed. Therefore, there are a large number of beam alignment measurements that must be performed along the NIF beam line to assure that the pointing and alignment control system centers the beam in order to provide the maximum energy on the fusion target located in the associated chamber. ${ }^{1-3}$ An automatic alignment (AA) system was designed and implemented to assure successful deployment of the high energy beam in each of the 192 beam lines. However, since a variety of techniques are provided to perform the alignment, there is a quantifiable uncertainty associated with each technique that may or may not meet the desired accuracy and precision specifications associated at each control point. Therefore, there is a need for a post-processing technique which accepts as input an uncertain position measurement and provides as output an improved position estimate (see Fig. 1).

Perhaps the most challenging of all beam line measurements are those made on the KDP (potassium dihydrogen phosphate) crystals. These crystals are critical elements used to double or triple the frequency of the laser beam as it passes providing a shorter wavelength. The higher frequency determined from the target physics enables laser plasma interactions for fusion. The NIF final optics assembly (FOA) matches lenses to this particular frequency producing the beam that is tightly focused on the target capsule required to achieve fusion ignition. In order for the KDP crystals to optimally double or triple the laser operating frequency, they must be precisely positioned at the appropriate angle. This is one of the critical tasks of the AA system. The KDP measurement consists of using a charge coupled device (CCD) imaging camera which produces a noisy back reflection image of a diagnostic test beam. The noise is caused by the camera itself as 
well as uncertainties that are due to small pointing errors made during the measurement. A sophisticated two-dimensional (2D) phase-only, matched-filter ${ }^{4}$ algorithm was developed to provide the initial raw position estimates for our model-based scheme. The advantage of the model-based technique in this application is that it enables us to decompose the noise from the process (sometimes called boiling noise) as well as the actual measurement.

The image is acquired from the CCD imaging camera produces both noisy measurement and reference images. A precise reference image is used to provide the desired fiducial that is used by the alignment system. Corrections to align the measured image with the reference is accomplished using the dedicated control loops that adjust pointing mirror stepping motors until the deviations between both reference and measured positions are within acceptable limits. ${ }^{3}$ Ultimately, the goal is to make this difference zero assuring proper beam alignment. Fig. 2 shows a set of measured KDP images with position estimates along with the reference measurement made from a very precise device. Note how tightly clustered the reference positions are compared to the raw measurements indicating its high precision. The objective, therefore, of the control loop is to position the beam mirrors such that these positions completely overlap (zero deviation) as shown in the bottom of the figure. Thus, the smaller the $x y$-deviations, the closer the beam is to the centerline reference assuring a tightly focused, high energy beam on target---the goal of the alignment system.

Should for some reason an anomaly develops in any beam line, it may not be possible to align for a particular shot. The timely detection of beam line anomalies are necessary to avoid future problems, which if left unmitigated could result in less than optimum performance of the laser. In this work, we show how a model-based processor (MBP) can be used to detect anomalies, on-line, during the calibration phase of a laser shot. This is an important by-product of the model-based approach in this application.

Thus, we discuss the feasibility of applying model-based processors as an on-line post-processing technique to improve the final position estimates provided by a variety of centroid based estimators and detect anomalies in a high energy laser beam line. These estimators are used to align high powered laser beams for experiments at NIF. In this paper we first motivate a stochastic model of the overall process and measurement system in Sec. 2. Here we show that a Gauss-Markov formulation is quite reasonable. Once this is complete, we discuss the underlying model-based theory for both position estimation and anomaly detection in Sec. 3. With the theory in hand, we develop the processor for the KDP application in Sec. 4 through ensemble statistics, simulation and eventual application to real measurement and reference data. Finally we conclude our results and point to future work in the final section. 


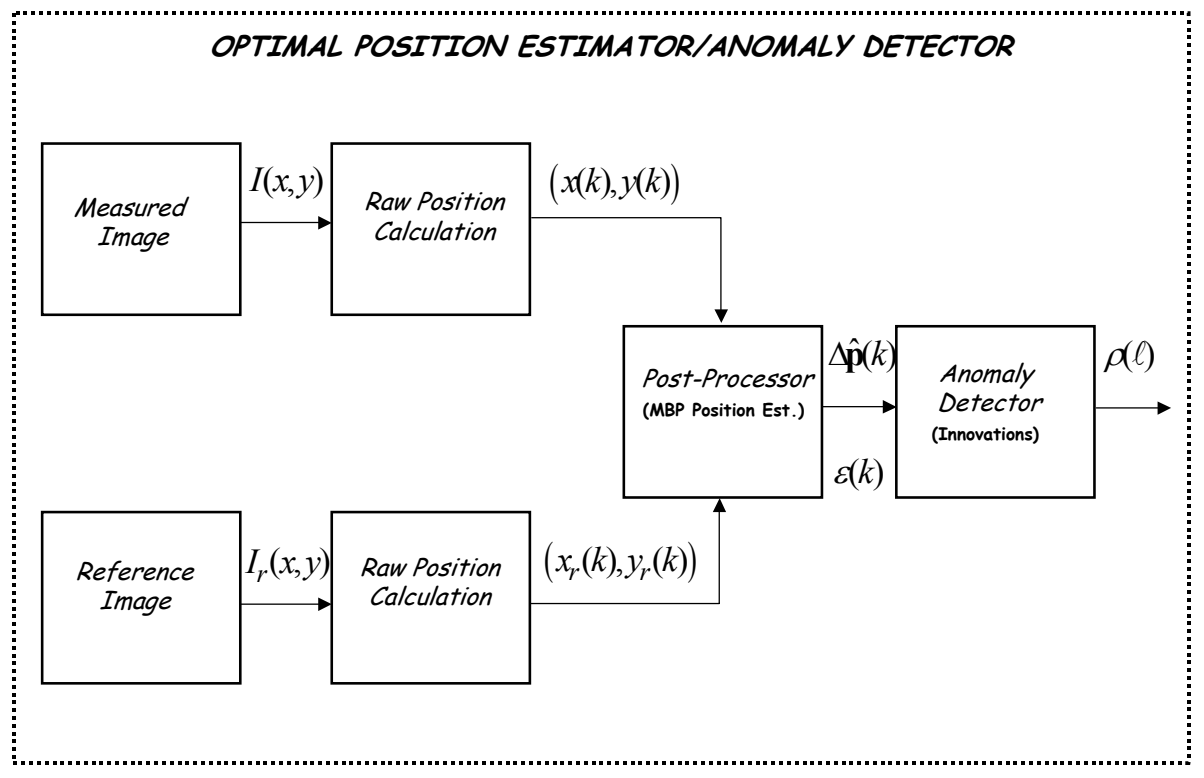

Fig. 1. Optimal position estimation with anomaly detector: Model-based post-processing and innovations-based anomaly detection.

\section{STOCHASTIC MODELING OF POSITION MEASUREMENTS}

The typical beam position estimator is accomplished by calculating the centroid of the measured images, that is, the centroid estimator is determined by the calculating the normalized position means given by

$$
\hat{x}=\frac{\iint x \operatorname{Pr}(x, y) d x}{\iint \operatorname{Pr}(x, y) d x d y} ; \quad \hat{y}=\frac{\iint y \operatorname{Pr}(x, y) d y}{\iint \operatorname{Pr}(x, y) d x d y},
$$

where $\operatorname{Pr}(x, y)$ is the joint probability density function. ${ }^{7}$ The point is that these measured positions are derived from the associated noisy CCD images. We define the true centroid positions by the position vector

$$
\mathbf{p}(k):=\left[\begin{array}{l}
\mathbf{x}(k) \\
\mathbf{y}(k)
\end{array}\right],
$$

where $\mathbf{p} \in \mathbf{R}^{N_{p} \times 1}$, and $k$ represents the $k^{\text {th }}$ sample (time). Since quite a number of images are acquired daily, a large data base consisting of position estimates are available, 
say, $P(k):=\{\mathbf{p}(k) ; k=1, \cdots, K\}$ and the position estimates are to be updated continuously. Since we know that the images are contaminated with noise and uncertainty, a more reasonable position measurement model is given by

$$
\mathbf{z}(k)=C \mathbf{p}(k)+\mathbf{v}(k),
$$

where $\mathbf{z}, \mathbf{p}, \mathbf{v} \in \mathbf{R}^{N_{z} \times 1}, C \in \mathbf{R}^{N_{z} \times N_{p}}$ and $\mathbf{v} \sim N\left(0, \mathrm{R}_{v v}\right)$, that is, the measurement noise is assumed zero mean, multivariate Gaussian with covariance matrix, $\mathrm{R}_{v v} \in \mathbf{R}^{N_{v} \times N_{v}}$.

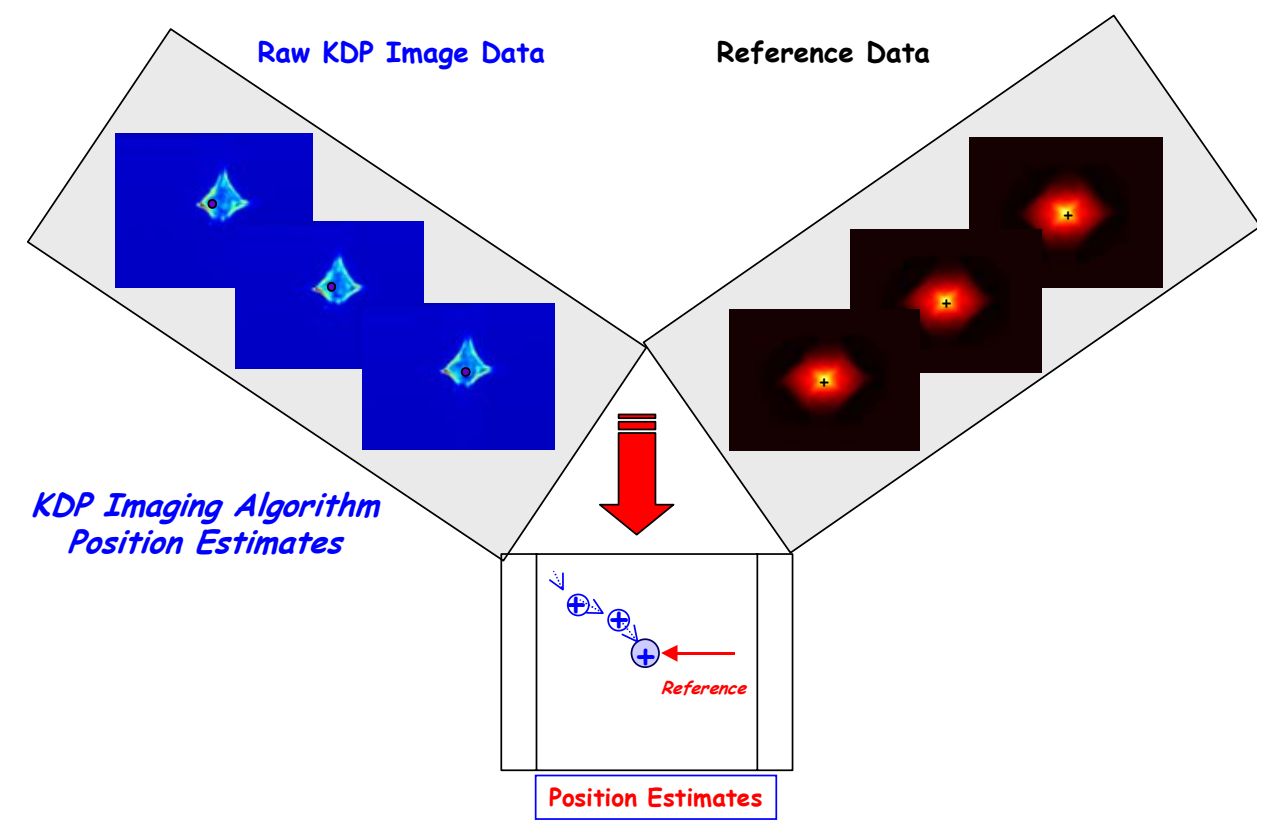

Fig. 2. Raw KDP crystal back-reflection image and reference position estimates.

We also note that since the CCD camera uses the identical beam line to measure both images, we model the position estimates as piecewise constants contaminated with beam line noise besides that noise contributed by the measurement systems. This process noise can be considered fluctuations caused by the inherent system optical transfer functions and turbulence caused by the argon gas filled housing during the laser beam propagation. Therefore, we assume that the contaminated position measurement is represented as

$$
\dot{\mathbf{p}}(t)=\mathbf{0}+\mathbf{w}(t) ; \mathbf{p}(0)=[x(0) \mathrm{y}(0)]^{T},
$$


in continuous time, but if we discretize over the $k$-sample images, then using first differences we obtain

$$
\dot{\mathbf{p}}(t)=\frac{\mathbf{p}\left(t_{k+1}\right)-\mathbf{p}\left(t_{k}\right)}{\Delta t_{k}}=\mathbf{w}\left(t_{k}\right) \text { for } \Delta t_{k}:=t_{k+1}-t_{k} \text {. }
$$

Substituting in Eq. (4) and assuming that $t_{k} \rightarrow k$, we obtain the following Gauss-Markov position model

$$
\mathbf{p}(k+1)=\mathbf{p}(k)+\Delta t \mathbf{w}(k)
$$

where $\mathbf{w} \in \mathbf{R}^{N_{p} \times 1}$ and $\mathbf{w} \sim \mathbf{N}\left(0, R_{w w}\right)$ along with the accompanying measurement model of Eq. (3).

$$
\mathbf{z}(k)=C \mathbf{p}(k)+\mathbf{v}(k),
$$

where $\mathbf{p}, \mathbf{w} \in \mathrm{R}^{N_{p} \times 1}, \mathbf{z}, \mathbf{v} \in \mathrm{R}^{N_{z} \times 1}$ for $C \in \mathrm{R}^{N_{z} \times N_{p}}$ and the uncorrelated noises are given by: $\quad \mathbf{w} \sim \mathrm{N}\left(0, \mathrm{R}_{w w}\right), \mathbf{v} \sim \mathrm{N}\left(0, \mathrm{R}_{v v}\right)$ with $\mathbf{p}(0) \sim \mathrm{N}\left(\overline{\mathbf{p}}(0), \overline{\mathrm{R}}_{\mathrm{pp}}(0)\right)$, the initial mean and associated covariance. This completes the basic description of the underlying GaussMarkov model. We note in passing that for a complete description of the general model including means, $\mathbf{m}_{p}(k), \mathbf{m}_{z}(k)$ with their associated covariances etc. (see Ref. 5 and 6 for more details).

Since we have both final measured and reference position estimates, we re-define a more convenient position vector by grouping the positions as

$$
\mathbf{p}(k):=\left[\begin{array}{c}
x(k) \\
y(k) \\
--- \\
x_{r}(k) \\
y_{r}(k)
\end{array}\right] \text { and } \mathbf{w}(k):=\left[\begin{array}{c}
w_{x}(k) \\
w_{y}(k) \\
--- \\
w_{x_{r}}(k) \\
w_{y_{r}}(k)
\end{array}\right]
$$

with the corresponding process noise covariance matrix given by 


$$
\mathrm{R}_{w w}=\left[\begin{array}{ccc}
\overline{\mathrm{R}}_{w w} & \mid & 0 \\
- & - & - \\
0 & \mid & \overline{\mathrm{R}}_{w_{r} w_{r}}
\end{array}\right],
$$

since each of the measured and reference images are uncorrelated.

This type of formulation enables us to estimate the process noise covariances independently for each image as well as characterize their uncertainties individually. Since the control loop jointly uses both the reference and measured images to produce its final centroid position estimates, we model the measurement matrix as the deviation (difference) between these data contaminated with independent measurement noise, that is, our measurement model of Eq. (3) becomes

$$
\mathbf{z}(k)=\left[\begin{array}{cccc}
1 & 0 & -1 & 0 \\
0 & 1 & 0 & -1
\end{array}\right] \mathbf{p}(k)+\mathbf{v}(k)
$$

with measurement covariance matrix

$$
\mathrm{R}_{v v}=\left[\begin{array}{ccc}
\sigma_{v v}^{2} & \mid & 0 \\
- & - & - \\
0 & \mid & \sigma_{v_{r} v_{r}}^{2}
\end{array}\right],
$$

and $\sigma_{v v}^{2}=\sigma_{x x}^{2}+\sigma_{y y}^{2}$ and $\sigma_{v_{r} v_{r}}^{2}=\sigma_{x_{r} x_{r}}^{2}+\sigma_{y_{r} y_{r}}^{2}$. Note that the deviations are defined by

$$
\Delta \mathbf{p}(k):=C \mathbf{p}(k)=\left[\begin{array}{l}
x(k)-x_{r}(k) \\
y(k)-y_{r}(k)
\end{array}\right] .
$$

This completes the section on position (uncertainty) modeling, next we consider the development of the optimal position estimator.

\section{MODEL-BASED POSITION ESTIMATION/ANOMALY DETECTION THEORY}

It is well-known that the optimal solution to the position estimation problem under the Gauss-Markov model assumption is provided by the model-based (state-space) processor or Kalman filter. ${ }^{5,6}$ This solution can be considered a predictor-corrector design in which the processor uses the model (random walk) to predict in absence of a measurement and then corrects the estimate when the measurement becomes available. Under the assumption of a perfect model, that is, the model imbedded in the process 
exactly matches the process, the MBP is capable of achieving the optimal minimum (error) variance estimate.

For the position estimation problem, the MBP takes on the following predictorcorrector form:
Prediction:
$\hat{\mathbf{p}}(k+1 \mid k)=\hat{\mathbf{p}}(k \mid k)$
Innovation:
$\varepsilon(k+1)=\mathbf{z}(k+1)-\hat{\mathbf{z}}(k+1 \mid k)=\mathbf{z}(k+1)-C \hat{\mathbf{p}}(k+1 \mid k)$
Correction:
$\hat{\mathbf{p}}(k+1 \mid k+1)=\hat{\mathbf{p}}(k+1 \mid k)+G(k+1) \varepsilon(k+1)$
Gain:

$$
G(k+1)=\tilde{P}(k+1 \mid k) C^{T} R_{\varepsilon \varepsilon}^{-1}(k+1)
$$

for $\hat{\mathbf{p}}(k+1 \mid k):=E\{\mathbf{p}(k) \mid \mathbf{p}(k-1), \cdots, \mathbf{p}(1)\}$ and $\hat{\mathbf{z}}(k+1 \mid k):=E\{\mathbf{z}(k) \mid \mathbf{z}(k-1), \cdots, \mathbf{z}(1)\}$ the underlying conditional means and $\mathrm{G}(k+1) \in \mathrm{R}^{N_{z} \times N_{p}}$ is the corresponding gain matrix ${ }^{6}$ calculated by the corresponding error covariance matrix, $\hat{P}(k+1 \mid k)=\operatorname{Cov}(\tilde{\mathbf{p}}(k+1 \mid k))$ with $\tilde{\mathbf{p}}(k+1 \mid k):=\mathbf{p}(k+1)-\hat{\mathbf{p}}(k+1 \mid k)$. The innovations covariance matrix is defined by $R_{\varepsilon \varepsilon}^{-1}(k+1)$.

The optimal position estimation algorithm is summarized in Table 1. 
Table 1. Optimal Position Estimation Algorithm with Anomaly Detection

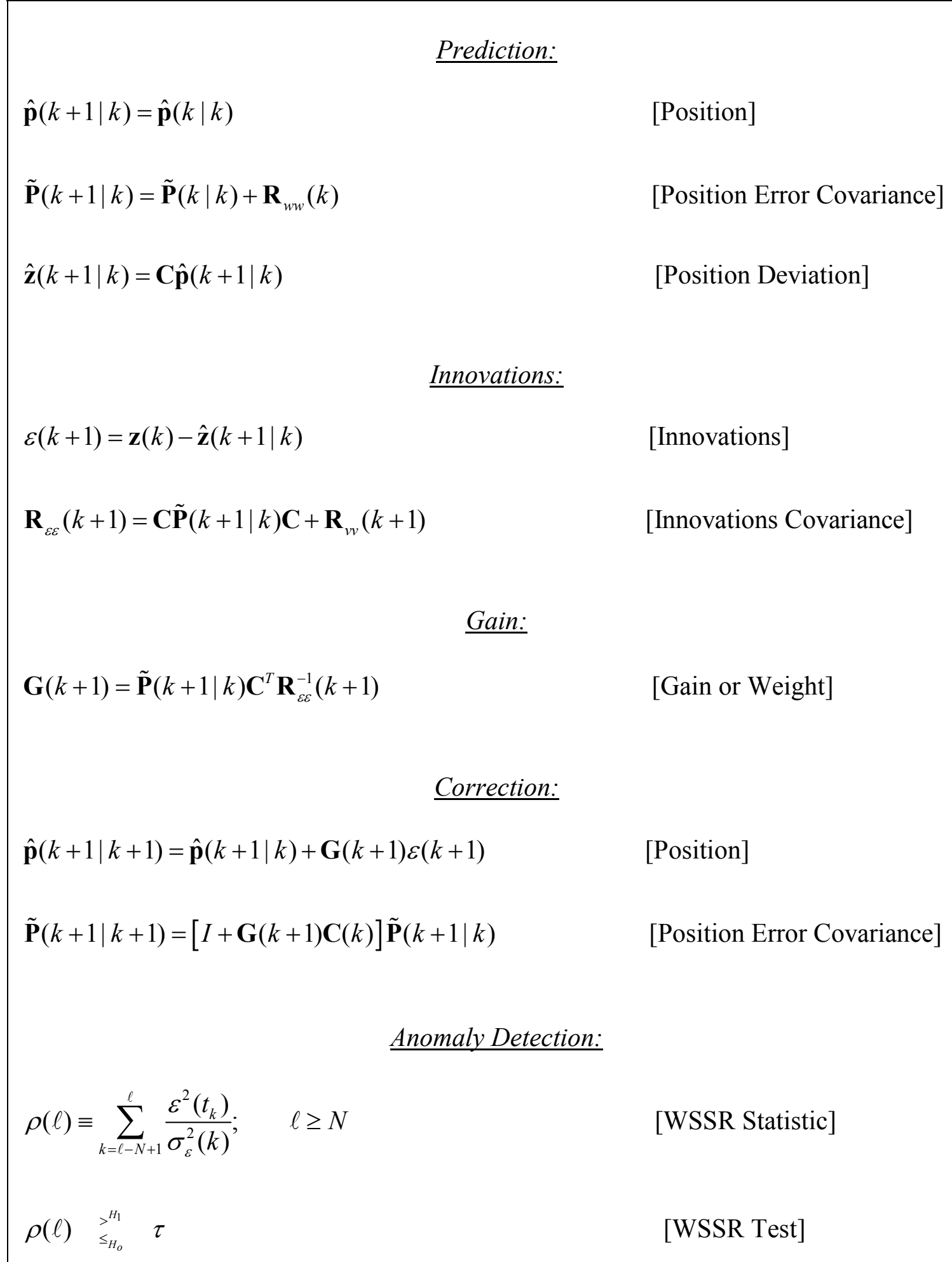


It is interesting to note that a necessary and sufficient condition for the MBP to be optimal is that the innovations sequence be zero mean and white (uncorrelated) --conditions that we test during processor design. It is possible to exploit this property of the innovations to detect anomalies in the system revealing potential problems in the beam line in pseudo real-time---a large asset when attempting to automate the alignment system. We develop two detection techniques that are used to monitor the position estimates from the daily measurements: whiteness detector and the weighted-sumsquared-residual (WSSR) detector. Both of these techniques rely on the assumption that the position deviations in the loop should change little during the daily measurement cycles. The underlying idea in anomaly detection is that the processors are "tuned" during calibration to operate in an optimal manner (innovations zero-mean/white). However, when an anomaly occurs, the innovations no longer maintain their optimal statistical properties leading to a "change from normal operation" and an anomaly is declared. With this detection, a decision must be made to either classify the anomaly or perform some other action. The main point to realize is that since we are using a recursive-in-time MBP, the innovations represent "how well the position model and its underlying statistics fit the raw data." If it is an optimal fit, then the innovations should be zero-mean and statistically white (uncorrelated) and the detectors evolve naturally.

The whiteness/zero-mean tests, that is, testing that $95 \%$ of the sample (normalized innovation) correlations lie within the bounds given by

$$
\left.\left\lceil\hat{c}_{\varepsilon \varepsilon}(k) \pm \frac{1.96}{\sqrt{N}}\right\rceil ; \quad \hat{c}_{\varepsilon \varepsilon}(k) \equiv \frac{\sigma_{\varepsilon}^{2}(k)}{\sigma_{\varepsilon}^{2}(0)}, \quad \text { [whiteness test }\right]
$$

and the corresponding zero-mean test defined by

$$
\left\lceil\hat{m}_{\varepsilon}(k)<1.96 \sqrt{\frac{\sigma_{\varepsilon}^{2}(k)}{N}}\right\rceil, \quad[\text { zero }- \text { mean test }]
$$

both rely on quasi-stationary assumptions and sample statistics to estimate the required correlations. However, it can be argued heuristically that when the MBP estimator is tuned, the nonstationarities are being tracked by the processor and therefore, the innovations should be covariance stationary.

When data are nonstationary, then a more reliable statistic to use is the WSSR which is a measure of the overall global estimation performance of the processor again determining the "whiteness" of the innovation sequence. ${ }^{6}$ It essentially aggregates all of the information available in the innovations and tests whiteness by requiring that the decision function lies below the specified threshold to be deemed statistically white. If

\footnotetext{
- Whiteness implies temporally uncorrelated samples along with an impulse-like autocorrelation (whiteness test) or equivalently a flat power spectrum.
} 
the WSSR statistic does lie beneath the calculated threshold, then theoretically, the estimator is tuned and said to converge or in our problem there is no abrupt change. That is, for our raw position measurements, we test that the corresponding innovations sequence is zero-mean/white by performing a statistical hypothesis test against the threshold. The WSSR statistic is estimated over some finite window of $N$ samples, that is, the $W S S R$ statistic $\rho(\ell)$ is defined by

$$
\rho(\ell) \equiv \sum_{k=\ell-N+1}^{\ell} \frac{\varepsilon^{2}\left(t_{k}\right)}{\sigma_{\varepsilon}^{2}(k)} ; \quad \ell \geq N
$$

and the detection algorithm is

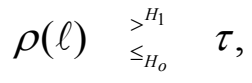

where $H_{o}$ is the hypothesis that the model "fits" the data (white innovations), while $H_{1}$ is the hypothesis that there is a mismatch or change specified by non zero-mean, non-white innovations. Under the zero-mean assumption, the WSSR statistic is equivalent to testing that the innovations sequence is white. Under $H_{o}, W S S R$ is distributed $\chi^{2}(N L)$. It is possible to show ${ }^{8}$ for a large $N L>30$ and a level of significance of $\alpha=0.05$, the threshold is given by

$$
\tau=N L+1.96 \sqrt{N L}
$$

Here the window is designed to slide through the innovations data and estimate its whiteness. Even in the worst case where these estimators may not prove to be completely consistent, the processor (when tuned) predicts the nonstationary innovations covariance, $\sigma_{\varepsilon}^{2}(k)$ enabling a simple (varying with $\ell$ ) confidence interval to be constructed and used for testing similar to Eq. (12) above. Thus, overall performance of the processor can be assessed by analyzing the statistical properties of the innovations. This completes the description of the MBP and the associated anomaly detectors, next we consider a simulation of the data and processor to predict the expected performance.

\section{POSITION ESTIMATION/ANOMALY DETECTION FOR THE KDP BEAM LINE}

In this section we discuss the application of optimal position estimators based on daily historical position estimates (data base) provided by the KDP algorithm. The data are a record of 48 days of final positions output from both the accurate reference system (reference data) and the estimated final position output from the KDP back-reflection data coupled to a phase-only matched filter ${ }^{4}$ imaging algorithm. The motivation for applying the model-based approach to this data set is to obtain a more accurate and precise estimate of error deviations from the reference characterizing the overall control loop performance in that position of the beam line. Theoretically, the position deviations 
between the reference and the KDP estimates should ideally be zero, but because of the beam line noise and variations, CCD camera limitations and control system tolerances, this is not the case. Instead the overall error statistics are used to bound the performance and assure that they remain within design specification from the error budget to achieve the overall project success (ignition). A scatter plot of the xy-positions from the reference (circles) and the raw KDP back reflection position estimates are shown in Fig. 2. Even though the actual estimates are grouped into two separate clusters due to improvements in the beam line over the 48 day period, the deviations between them should tend to cluster about zero.

\section{MBP Design}

In the design of the MBP the usual procedure is to: (1) develop the required models; (2) simulate a set of position data characterized by any of the a-priori information available; (3) develop the minimum error variance design; and (4) apply the processor to the available data set evaluating its performance.

We developed the basic model set assuming a Gauss-Markov structure as in Sec. II . These parameters of this model were estimated from the statistics of a large ensemble (> 5000) of images and performance statistics of the KDP centroiding algorithm. We used this information to construct the initial simulation of the MBP to "match" with the historical data available (48 days) and the corresponding ensemble statistics.

Thus, our approach is to first perform a simulation of the measurement process using estimated statistics from the data and then apply it to the actual data. During the simulation phase, we are able to analyze the performance of the MBP and assure ourselves that all of the models and statistics are correct. We expect to obtain the minimum variance estimates, if not, then it is usually an implementation issue. Once the simulation and model adjustments have been made, we apply the processor to the measured data.

\section{Gauss-Markov Simulation}

Now that we have developed the models and have some estimates from the ensemble statistics of the data base, we are now able to perform a Gauss-Markov simulation to assess the feasibility of the MBP. We simulated a set of data based on the following parameters using the mean XY-position estimates, that is, $x_{i}=\mu_{x_{i}} \pm 1.96 \sigma_{i}$ for both the

measured and reference data: $\mathbf{x}(0)=[344 \pm 8,270 \pm 13.8,344 \pm 7.8,270 \pm 14.4]^{\prime}$. We used the mean values as the initial position estimates with low error variances $\left(1 \times 10^{-6}\right)$. We chose an uncorrelated measurement (deviation) noise covariance matrix as: $R_{v v}=\operatorname{diag}[3.8$ 3.9]. The process noise covariance selected is 


$$
R_{w w}=\left[\begin{array}{cc}
R_{w w}(1) & 0 \\
0 & R_{w w}(2)
\end{array}\right] \text { for } R_{w w}(1)=\left[\begin{array}{cc}
0.01 & -0.0053 \\
-0.0053 & 0.01
\end{array}\right] ; \quad R_{w w}(2)=\left[\begin{array}{cc}
0.01 & -0.00753 \\
-0.00753 & 0.01
\end{array}\right]
$$

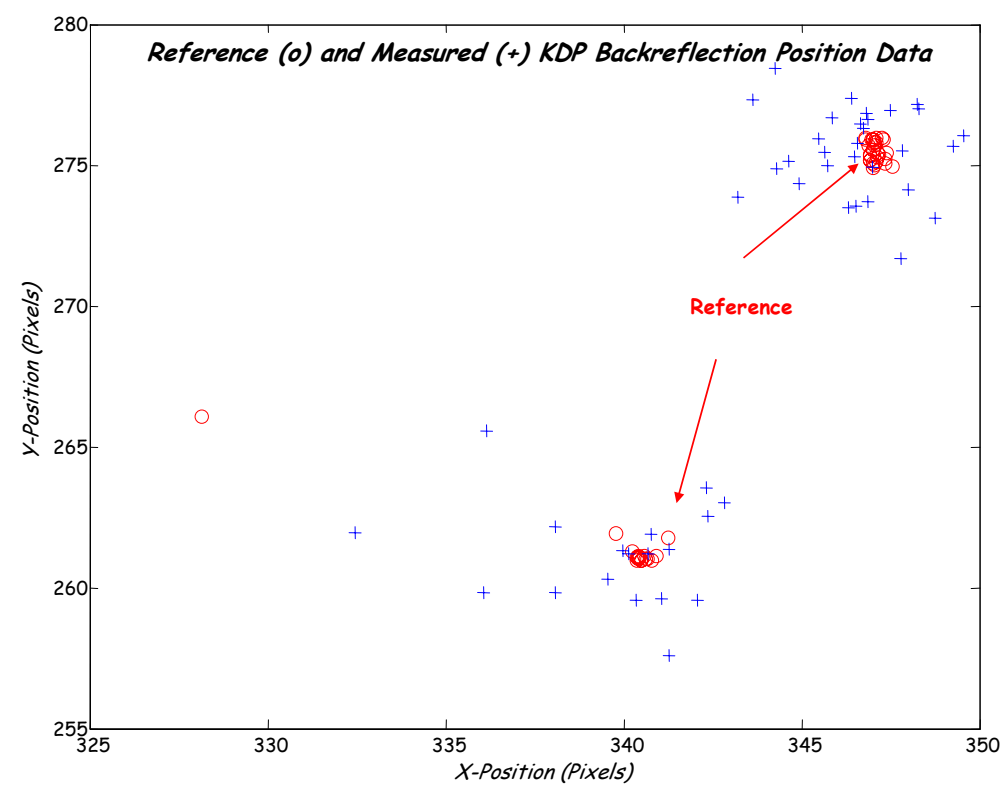

Fig. 3. Scatter plot of reference (O) and raw KDP position estimates $(+)$.

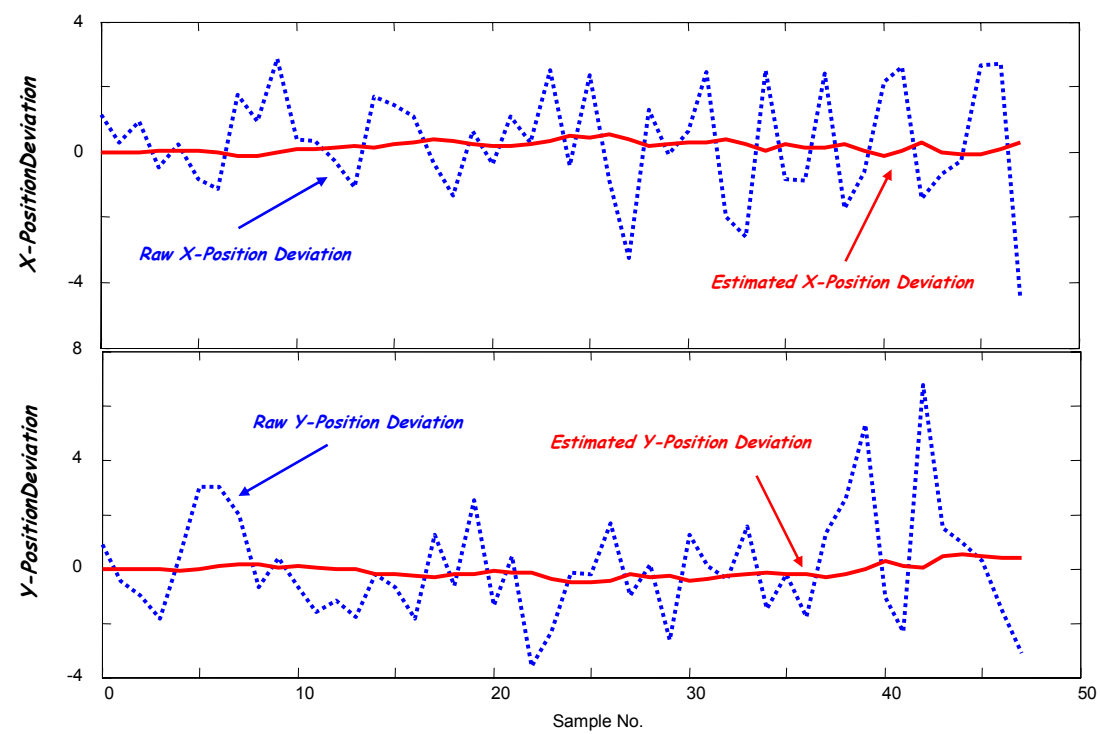


Fig. 4. Simulated XY-position deviations: Raw (dotted) and estimated (solid).

The synthesized positions have small variations due to the process noise, but are essentially constants (mean values). The simulated noisy $D X, D Y$-deviation measurements (dotted line) including the process and measurement noise are shown in Fig. 4. Next we executed the MBP over this data set and the position deviations (solid line) are also shown in the figure. Here we see that much of the raw measurement and process noise have been removed by the processor and only the deviation errors are shown. Recall that they should be close to zero for the alignment control system to be operating efficiently. To get a better feel for the overall improvement contributed by the MBP, we can compare the scatter plots of the raw and processed (estimated) deviations. We calculated the corresponding two-sigma covariance (error) ellipsoids ${ }^{8}$ that bound $95 \%$ of the deviations. These ellipsoids should be centered at zero $(0 D X, D Y)$ and the major and minor axes should be as small (better precision) as possible. Clearly from Fig. 5 , we see that the estimated deviations satisfy this constraint much better than the raw position deviations.

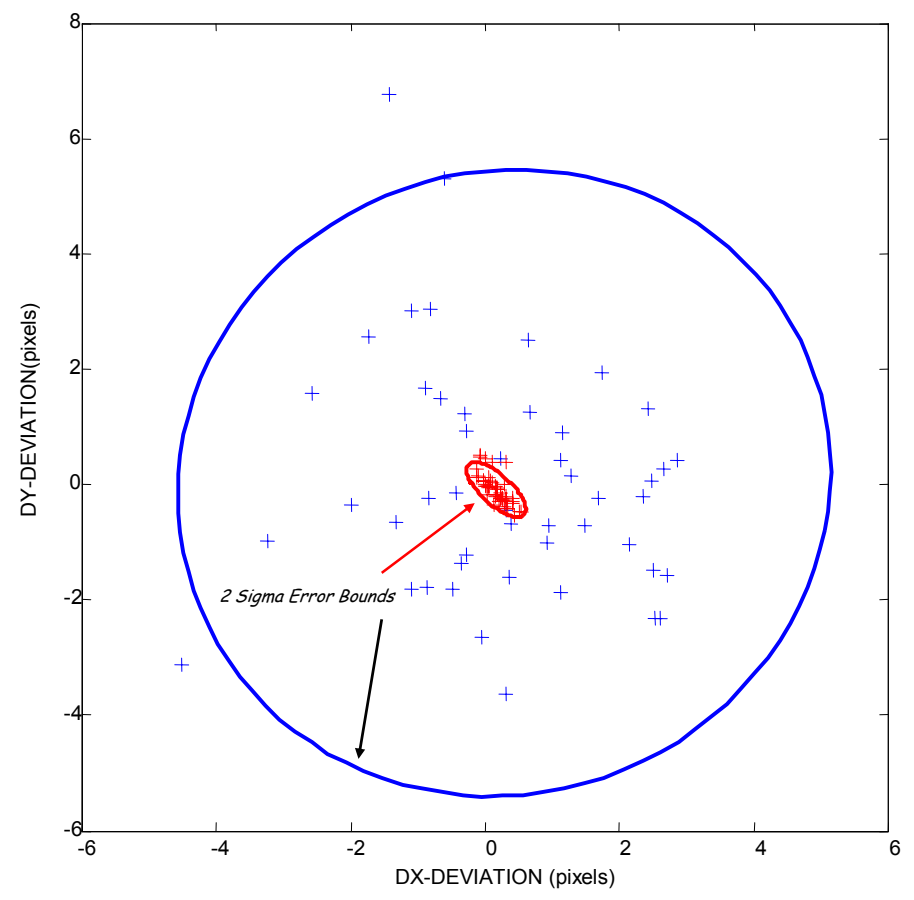

Fig. 5. Two sigma error ellipsoid for XY-position deviations from simulated data: Raw deviations (larger) and estimated (smaller). 
To validate the results, we investigate the minimum variance design procedure. We show the innovations sequences in Fig. 6a associated with each deviation along with their corresponding zero-mean/whiteness tests in $6 b$ and the WSSR test in $6 c$. It is clear from the figure that both sequences are zero-mean: $(0.12<0.68 ; 0.11<0.80)$; white: $(4 \%$ out; $0 \%$ out); and WSSR decision function lies below the threshold for a window of size $N$ : (Threshold $=69.6, N=25)$. These statistics indicate that the minimum variance position deviation estimates have been achieved. This result is expected, since the models used in the MBP are identical to those in the Gauss-Markov simulator. However, the results can be interpreted as the "best" (minimum variance) one could hope to do under these circumstances. Next we apply the MBP to the actual deviation data.

(a)

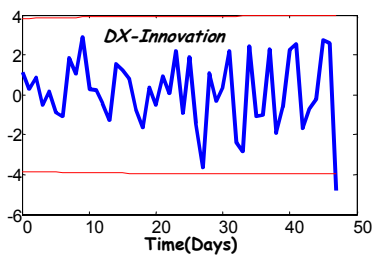

(b)

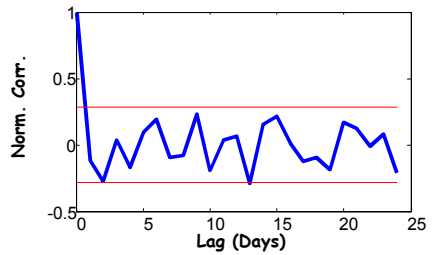

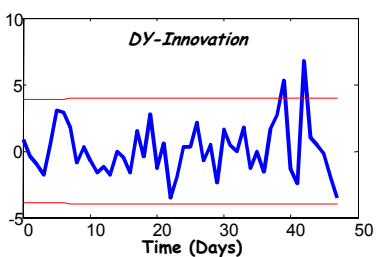

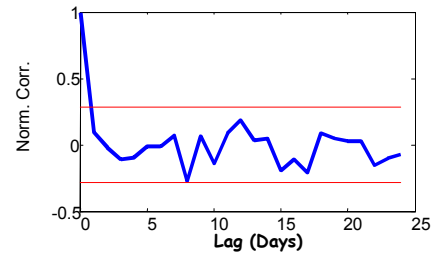

(c)

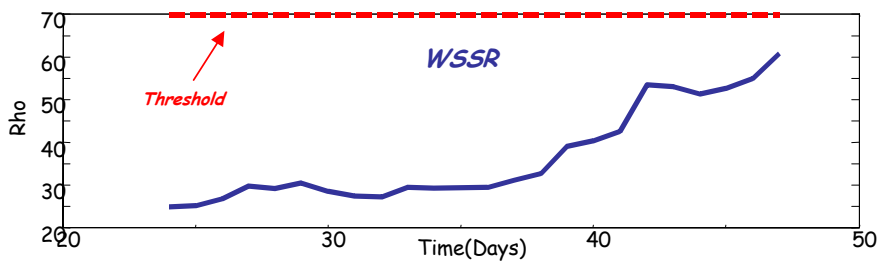

Fig. 6. MBP design for XY-position deviations: (a) Innovations for X-deviation and $\mathrm{Y}$ deviation. (b) Optimality tests: Zero-mean $(0.12<0.68 ; 0.11<0.80)$ and whiteness $(4 \%$ out; $0 \%$ out) (c) WSSR Test (threshold=69.6, $\mathrm{N}=25$ ).

\section{KDP Deviation Data}

In order to process the KDP deviation data, we must develop parameters for the underlying model embedded in the processor. We start with guesses of the noise (process and measurement) statistics from the simulator and then adjust them accordingly. For instance, if the innovation sequence lies outside its predicted bounds implying that the measurement noise variance is too small, then it can be adjusted to satisfy this constraint (see Ref. 6 for more details) and "match" the data. The process noise is actually quite difficult to fit, since it is directly proportional to the MBP gain. In essence, once all of the other model parameters are reasonably adjusted, they are then held fixed and the process 
noise covariance is varied to achieve the "best" possible (minimum error variance) innovations statistics (zero-mean/white, WSSR below $\tau$ ).

The results of the MBP design for the deviation data are shown in Figs. 7-10. We see the raw KDP position deviation estimates along with the optimal processor results over the 48 day period in Fig. 7. It is clear that the processor is tracking the trends in the data while reducing the noise or equivalently enhancing the SNR. To confirm this we observe the two-sigma error ellipsoid plots of Fig. 8 where we observe that much of the uncertainty has been removed and the estimated deviations are clearly clustered (centered) around the $(0,0)$ position and have a much smaller ellipsoid (better precision) than the raw data. Again to confirm the optimality of the processor we check the zeromean/whiteness/WSSR statistics (see Fig. 9) which give the following results. It is clear from the figure that both sequences are zero-mean: $(0.2 .2<0.71 ; 0.1<0.69)$; white: $(0 \%$ out; $0 \%$ out); and WSSR decision function lies below the threshold for a window of size $N$ : (Threshold $=69.6, N=25)$. These statistics again indicate that the minimum variance design has been achieved and the processor along with its associated statistics are valid and optimal. With this in mind we can also observe the performance of the MBP in estimating (filtering) both measured KDP and reference positions. The results are shown in Fig. 10. Here we see the estimated (filtered) positions observing that they are random (conditional mean) and vary slightly from the initial means indicating that the process noise is not too severe in this case. This completes the MBP design for the position estimation problem. Next we investigate the feasibility of using this approach for anomaly detection. 

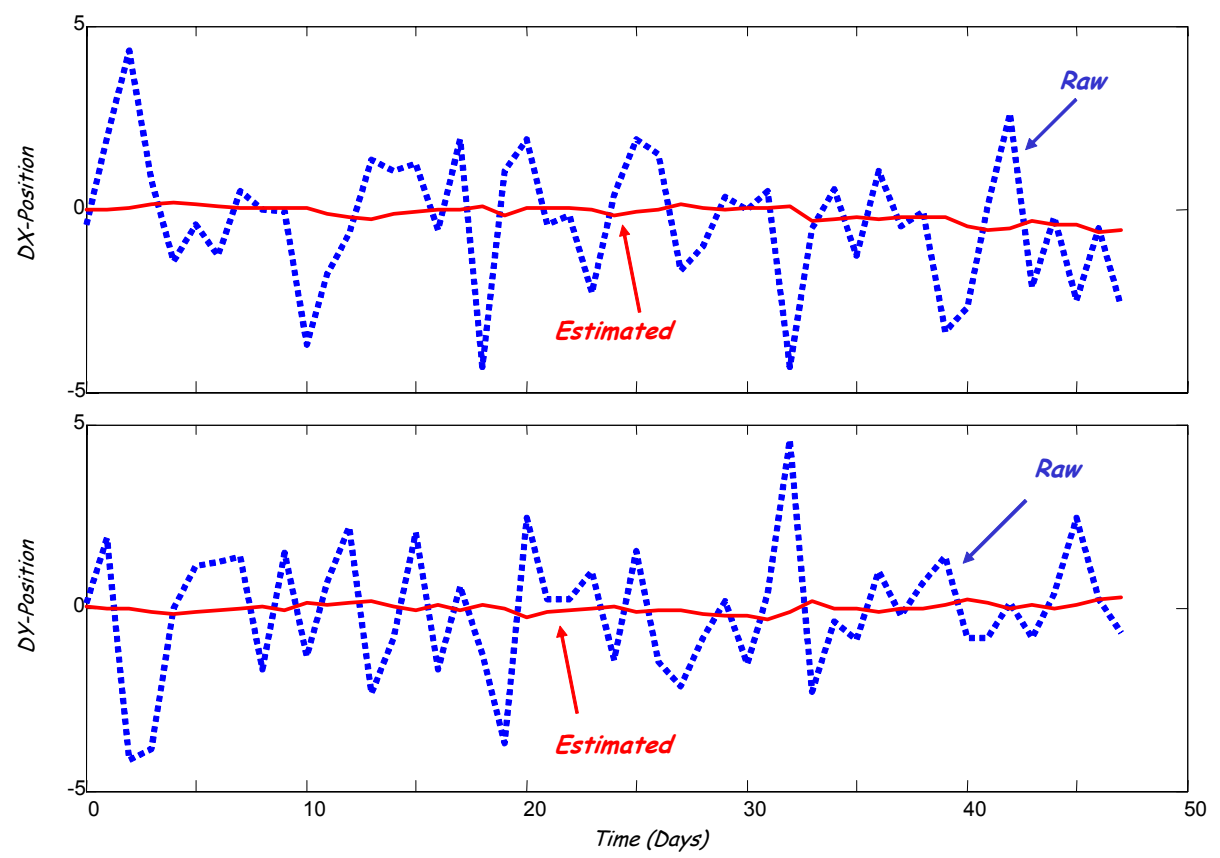

Fig. 7. Actual KDP XY-deviations: Raw (dotted) and estimated (solid).

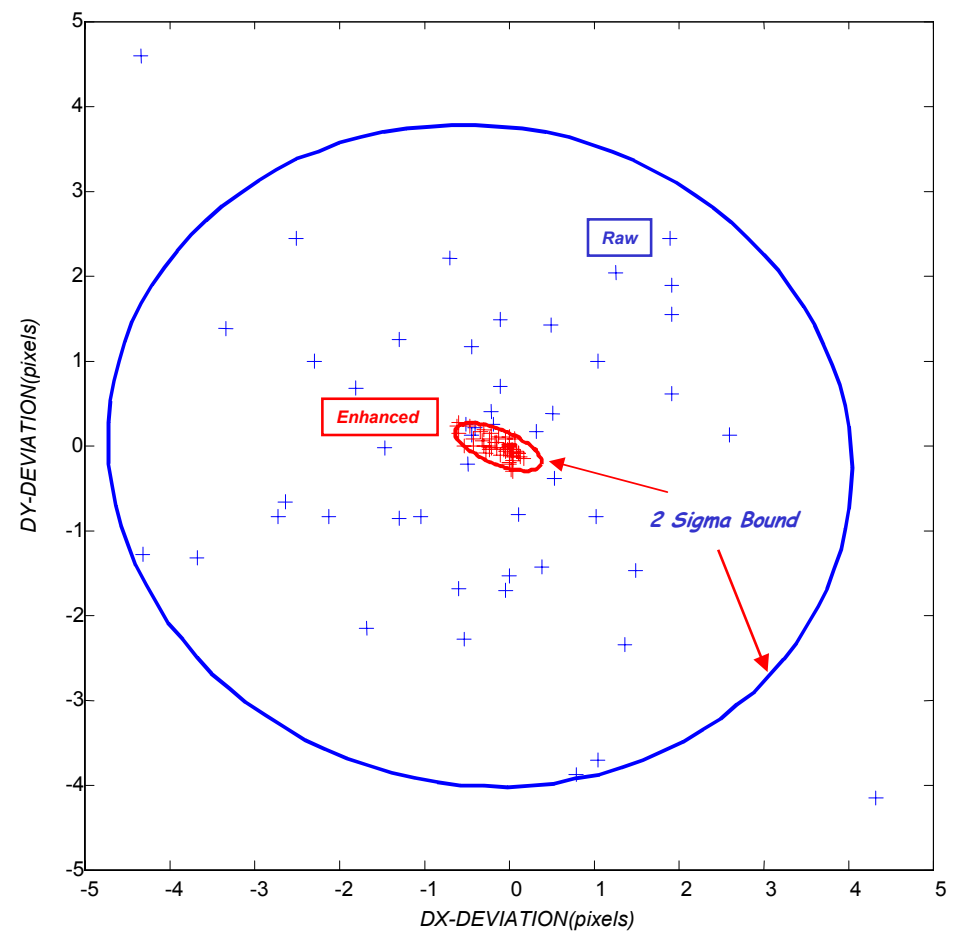

Fig. 8. Two sigma error ellipsoid for XY-position deviations from data: Raw deviations (larger) and estimated (smaller). 
(a)
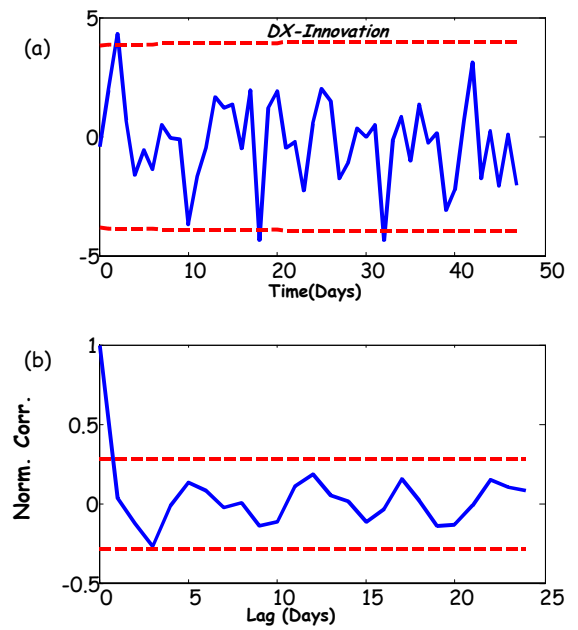

(c)

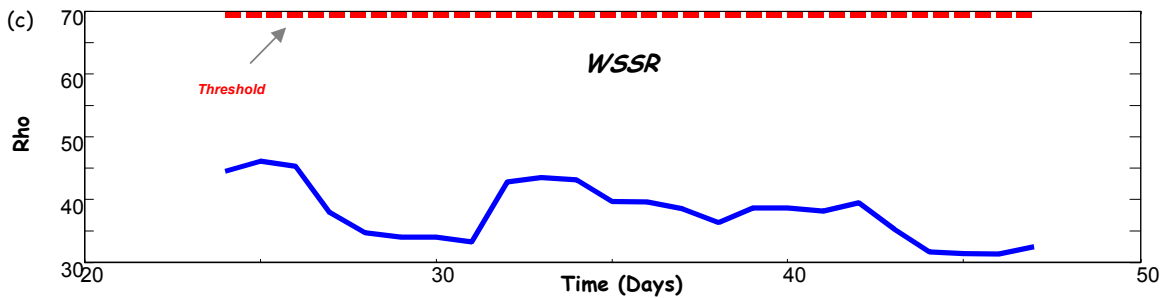

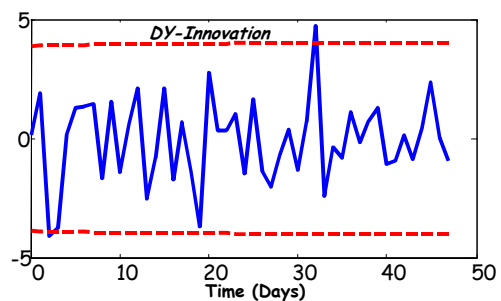

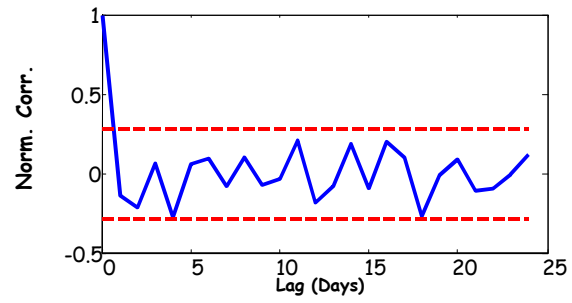

Fig. 9. Actual MBP design for XY-position deviations: (a) Innovations for $\mathrm{X}$-deviation and Y-deviation. (b) Optimality tests: Zero-mean $(0.2 .2<0.71 ; 0.1<0.69)$ and whiteness ( $0 \%$ out; $0 \%$ out) (c) WSSR test (threshold $=69.6, \mathrm{~N}=25$ ).

(a)

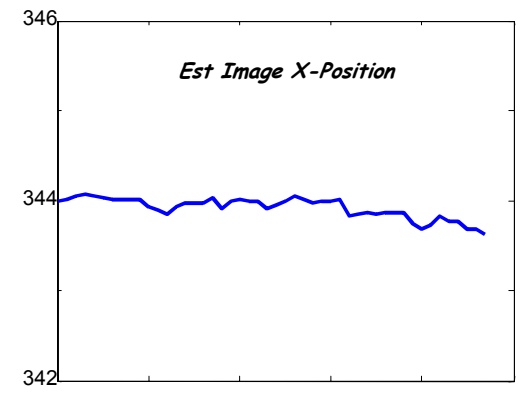

(b)

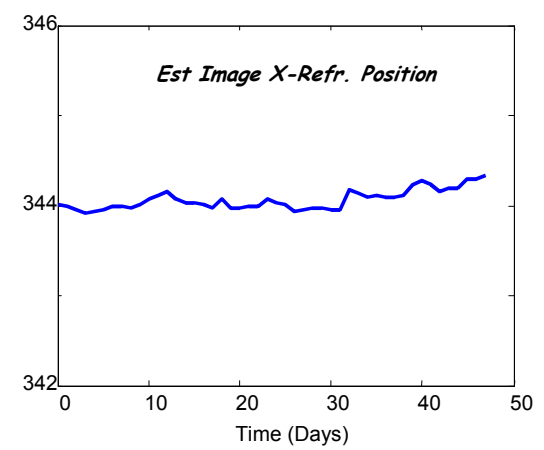




\section{Anomaly Detection Feasibility Study}

For this final investigation, we return to the simulation to assess the feasibility of applying the MBP as an effective means to detect anomalies (off-normal) that could occur in the beam line. Here we assume that the MBP has been "tuned" to normal operations for the particular beam line and optics. Thus, the innovations are zero-mean, white and the WSSR lies below the threshold for optimal design. To "simulate" an offnormal condition (in terms of position estimates), we increase the amplitude of both $\mathrm{X}$ and $\mathrm{Y}$ deviations tenfold during the period of 29-35 days. This can be thought of as corresponding to a pulse-like transient in either or both of the measurement and/or reference position data. We applied the optimal (for no anomaly) MBP to the data to determine whether or not it could detect and track the unknown transient anomaly.

The results of the effect on the deviation measurements are shown in Fig. 11. We see the normal measurement and then the transient "jump" within the prescribed time period. It is also interesting to note that there is a slight, but corresponding disturbance in the estimated (filtered) measurement as well indicating that there is enough of a disruption to cause the MBP to try to track it. However, the MBP is not able to track the transient very well---the expected results. Next we observe the deviation innovation sequences output by the MBP in Fig. 12. We again observe the transient in both sequences, since the position estimates do not track it well enough. The zeromean/whiteness tests seem a bit too insensitive to the rapid change (6 samples) and do not dramatically detect it; however, the whiteness tests do indicate a non-white sequence (4\% out (borderline); $8 \%$ out (non-white)). On the other hand, the WSSR test clearly detects "change from normal" caused by the simulated beam line anomalies almost instantaneously indicating a feasible solution. This is again expected since the WSSR statistic $(\rho(\ell))$ can be tuned to transient disruption by selecting the appropriate window length. For this problem we chose: $(N=10$; Threshold=32.4). To verify the performance of the zero-mean/whiteness test we observe the estimated positions (states) in Fig. 13. Here we see that the MBP actually responds to such a high amplitude level change in the anomaly transient. The processor could easily be "tuned" to ignore the change of the states by decreasing the process noise variance. Finally, the scatter plots for this case are shown in Fig. 14: one for the optimal solution (no anomaly) and one for the solution with the anomaly. The two-sigma error ellipsoid for the case with an anomaly clearly indicates that something is wrong since the estimated deviation uncertainty (larger ellipsoid) is much larger than that of the raw data. This completes the feasibility study of applying a model-based anomaly detector. 

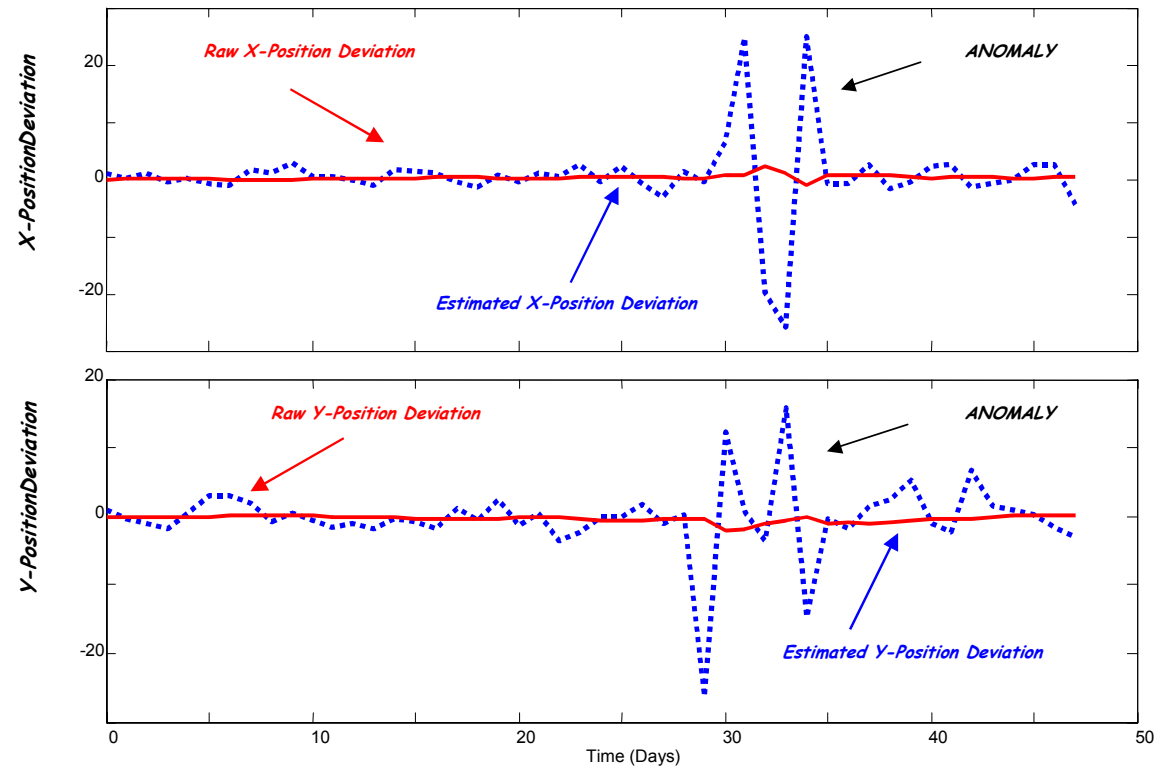

Fig. 11. Simulated XY-deviations with anomaly: Raw (dotted) and estimated (solid).

(a)
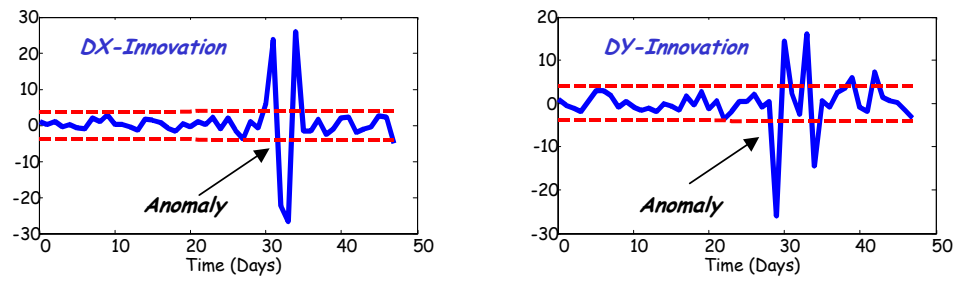

(b)
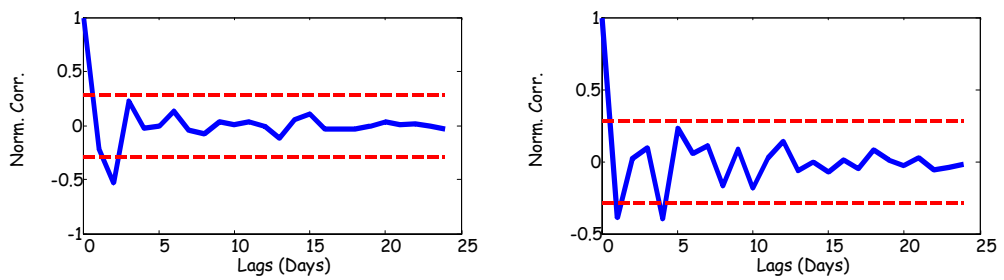

(c)

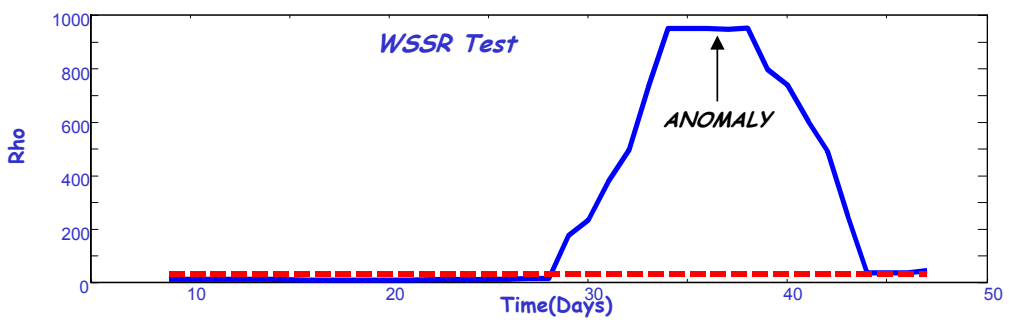

Fig. 12. MBP detection with simulated anomaly: (a) DX and DY innovations with anomaly. (b) Optimality tests: Zero-mean $(0.15<2.9 ; 0.03<2.2)$; whiteness (4\% out; $8 \%$ out). (c) WSST test (threshold=32.4; $\mathrm{N}=10$ ). 

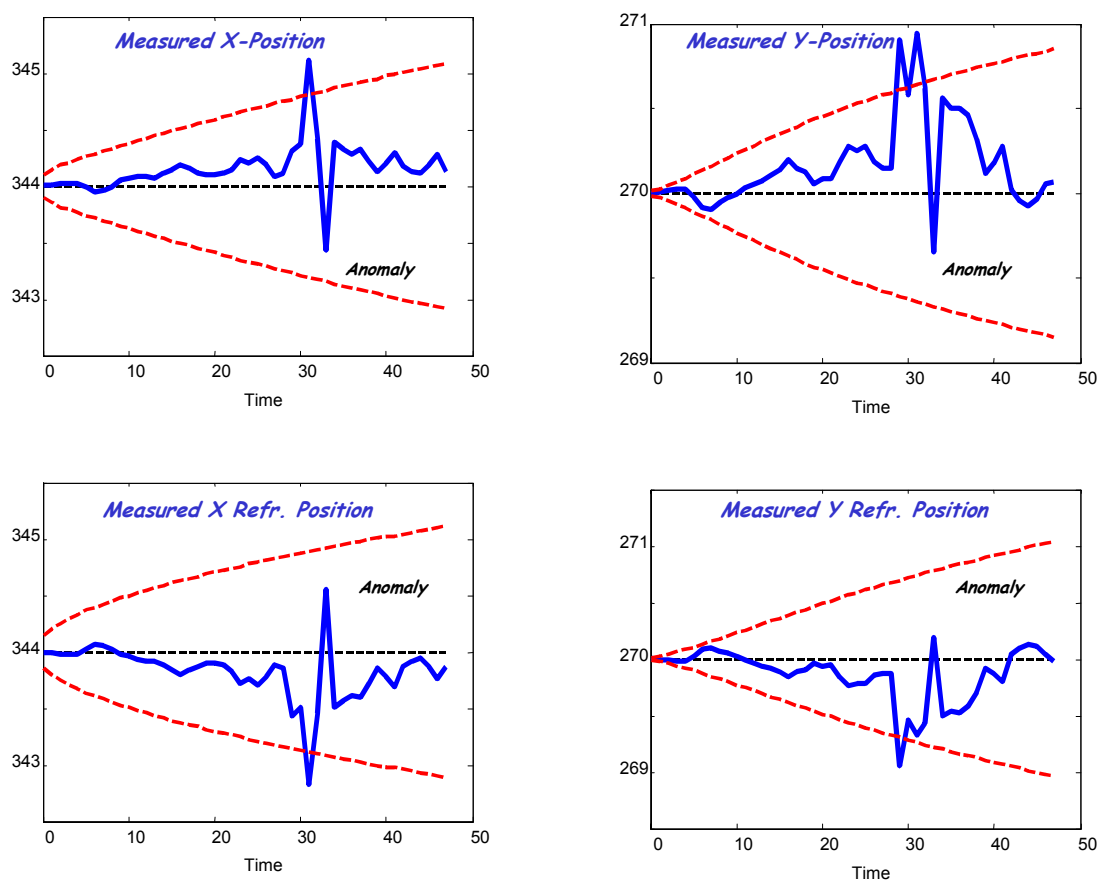

Fig. 13. Estimated XY-positions with anomaly: (a) Measured position estimates. (b) Reference position estimates.
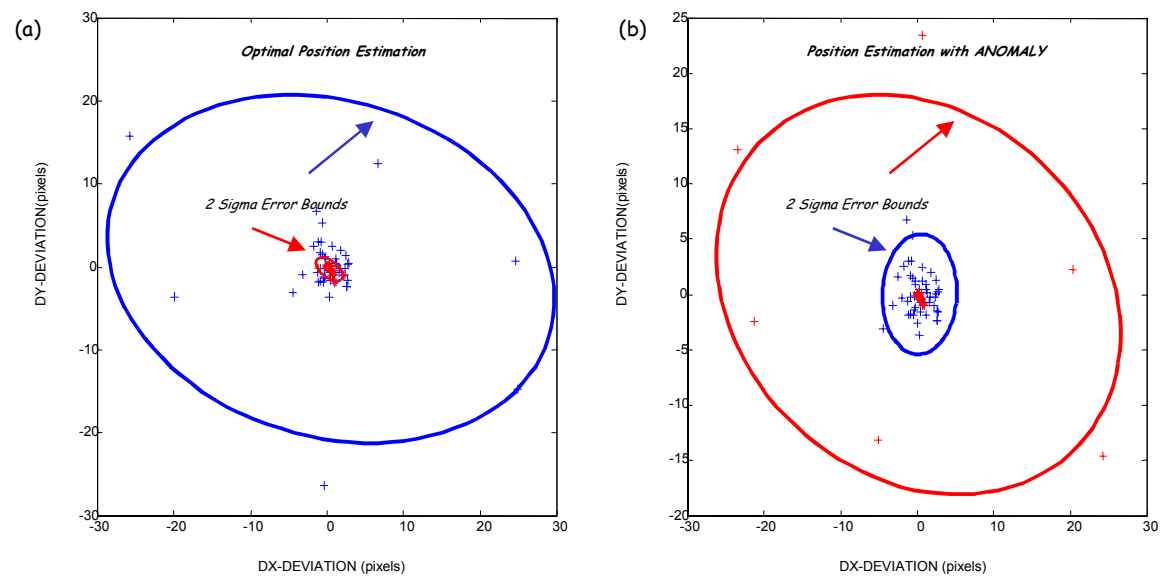

Fig. 14. Two sigma error ellipsoid and estimated XY-positions: (a) Measured data no anomaly. (b) Measured data with anomaly. 


\section{CONCLUSIONS}

The feasibility of apply model-based signal processing techniques to noisy beam position measurement data for the automatic alignment of a high energy laser has been demonstrated on both simulated and actual data sets. After motivating the need for an online post-processor capable of significantly improving position deviation estimates for automatic beam control as well as anomaly detection, we developed the required theory to define the problem in the Gauss-Markov framework leading to an optimal model-based processor (MBP) solution (Kalman filter). The design of the processor was accomplished using a third party toolbox in MATLAB ${ }^{9}$, SSPACK_PC. ${ }^{10}$ It was carefully orchestrated on both simulated and actual historical beam line data for the KDP crystal loop at the National Ignition Facility (NIF). It was shown that the minimum error variance design (zero-mean/white innovations) could be achieved for these data sets. The feasibility of using this approach coupled to an anomaly detector was clearly demonstrated on synthesized data. It was shown that even a rapid transient could be detected almost instantaneously.

Further work must proceed to validate the model-based approach performance on other beam lines. The on-line MBP should be incorporated into the actual control loops with a feedback gain to automate the alignment control system in attempting to achieve zero deviations. Daily data should be processed to obtain more realistic uncertainty measures relative to these position deviations under a wide variety of off-normal conditions to insure proper operation. In conclusion, we feel that the MBP can provide the mechanism towards achieving a truly automatic alignment system.

\section{ACKNOWLEDGMENT}

This work was performed under the auspices of the U. S. Department of Energy by University of California, Lawrence Livermore National Laboratory under Contract No. W-705-Eng-48.

\section{REFERENCES}

1. E. Moses, "The National Ignition Facility Comes to Life," Science \& Technology Review, LLNL Report, pp. 4-14, Sept. (2003).

2. F. R. Holderner, E. Ables, E. S. Bliss, S. J. Boege, R. D. Boyd, C. J. Chocol, D. T. Davis, R. D. Demaret, R. E. English, C. W. Laumann, J. L. Miller and S. W. Thomas, "Beam control and diagnostic functions in the NIF transport spatial filter," Proceedings of SPIE 3047, 692-699, (1997).

3. E. S. Bliss, F. R. Holderner, J. T. Salmon, J. R. Severyn, et. al., "Beam control and laser diagnostic systems," LLNL Report, UCRL-LR-105821-99-1, 79-97, (1999). 
4. A. Awwal, W. Mcclay, W. Ferguson, J. Candy, T. Salmon and P. Wegner, "Composite amplitude modulated phase-only filter based detection and tracking of the back-reflection of KDP images," in Photonic Devices and Algorithms for Computing VI, Proc. of SPIE 5556, (2004).

5. A. Jazwinski, Stochastic Processes, (Academic Press, New York,1970).

6. J. V. Candy, Signal Processing: The Model-Based Approach, (McGraw-Hill, New York, 1986).

7. K. R. Castleman, Digital Image Processing, (Prentice-Hall, New Jersey, 1979).

8. T. W. Anderson, An Introduction to Multivariate Statistical Analysis, (Wiley, New York, 1984).

9. Mathworks, MATLAB User's Manual,( Mathworks, Natick, MA, 1986)

10. J. V. Candy and P. M. Candy, "SSPACK_PC: Model-based processing on personal computers, DSP Applic., 2, (3), 33-42, (1993). 